\title{
ESTUDIO LECTINHISTOQUÍMICO DEL ÚTERO DE ALPACAS (Vicugna pacos) BAJO TRATAMIENTO SUPEROVULATORIO
}

\author{
Lectinhistochemistry Study of The Uterus of AlPaCAs (Vicugna pacos) \\ UNDER SUPEROVULATION TREATMENT
}

\author{
Verónica López C. ${ }^{1}$, María Vásquez C. ${ }^{1,5}$, Wilfredo Huanca L. ${ }^{2}$, Alexei Santiani A. ${ }^{2}$, \\ Claudio Barbeito ${ }^{3,4}$, Carolina Canuzzi A. ${ }^{3}$, Boris Lira M. ${ }^{1}$, José Rodríguez G. ${ }^{1}$
}

\section{Resumen}

El objetivo del presente estudio fue determinar el patrón de glicosilación del endometrio uterino en alpacas con y sin tratamiento de superovulación. Se utilizaron cinco alpacas no superovuladas con folículos ováricos menores de $4 \mathrm{~mm}$ y cinco alpacas que fueron sometidas a un tratamiento de superovulación en base a FSH, GnRH y hCG. Se tomaron muestras del cuerpo del útero y de ambos cuernos uterinos y se trabajaron con las técnicas histoquímicas PAS y Azul Alcián y con la técnica de lectinhistoquímica. Para esta última se utilizaron 14 lectinas, de las cuales PNA, RCA-I, ConA, DBA, WGA, sWGA, GS, LCA, SJA y PHA-L presentaron un patrón de glicosilación diferente entre ambos grupos, sugiriendo que el tratamiento hormonal influenció en la expresión de los glicoconjugados que se unen a estas lectinas. Asimismo, las lectinas PNA, SJA, SBA, ConA, LCA y PSA presentaron diferencias entre el cuerno derecho y el cuerno izquierdo, lo que sugiere que los glicoconjugados de unión a estas lectinas podrían participar en generar un ambiente propicio para la implantación, que por lo general se da en el cuerno izquierdo en la alpaca. Se concluye que la alpaca tiene un patrón de glicosilación que puede variar con tratamientos hormonales de superovulación.

Palabras clave: alpaca, lectinas, patrón de glicosilación, endometrio uterino, superovulación

\section{Abstract}

The aim of this study was to determine the glycosylation pattern in the uterine endometrium of alpacas with and without superovulation treatment. Uterine tissues were obtained from five non-treated alpacas with ovarian follicles less than $4 \mathrm{~mm}$ and

${ }^{1}$ Laboratorio de Fisiología Animal, ${ }^{2}$ Laboratorio de Reproducción Animal, Facultad de Medicina Veterinaria, Universidad Nacional Mayor de San Marcos, Lima, Perú

${ }^{3}$ Instituto de Patología, ${ }^{4}$ Cátedra de Histología y Embriología, Facultad de Ciencias Veterinarias, Universidad Nacional de La Plata, Argentina

${ }^{5}$ E-mail: evasquezc@gmail.com

Recibido: 1 de febrero de 2012

Aceptado para publicación: 12 de octubre de 2013 
five superovulated alpacas where FSH, GnRH and hCG was used. Samples from the uterus and uterine horns were analyzed by histochemistry (PAS and alcian blue) and lectinhistochemistry techniques. Fourteen lectins were used and PNA, RCA-1, ConA, DBA, WGA, Swga, GS, LCA, SJA and PHA-L showed different glycosilation patterns in the endometrium between both groups, indicating that the hormonal treatment could affect the expression of glycoconjugates that have affinity for these lectins. Lectins PNA, SJA, SBA, ConA, LCA and PSA showed different glycosilation patterns between the two uterine horns, indicating that the glycoconjugates that have affinity for these lectins can be implicated in the uterine environment during the implantation, which in most cases in the alpaca take place on the left horn. In conclusion, the glycosylation pattern in the alpaca can change due to superovulation hormone therapy.

Key words: alpaca, lectins, glycosylation pattern, endometrium, superovulation

\section{INTRODUCCIÓN}

La producción de alpacas en el Perú es de gran importancia socioeconómica (Montes et al., 2008; Quispe et al., 2009). Una de sus principales limitantes es la baja tasa de preñez, la cual está directamente relacionada con una alta tasa de mortalidad embrionaria (Novoa, 1991), que puede llegar al 50\% dentro de los primeros 35 días de gestación (Fernández-Baca et al., 1970).

Una de las causas de mortalidad embrionaria puede ser la inadecuada implantación del blastocisto en el útero, ya que se sabe que durante el proceso de implantación se requiere un estrecho contacto entre el trofoblasto del blastocisto y el glicocáliz del epitelio uterino (Tachi et al., 1970). Whyte y Allen (1985) comprobaron la abundancia de glicanos o polisacáridos, tanto en las superficies uterinas como en las del trofoblasto; además, se sabe que los glicoconjugados participan en varias funciones reproductivas, incluyendo el momento de aposición y adhesión del embrión al útero (Psychoyos, 1986).

En el ratón, cerdo, asno, caballo y camello se conoce que la presencia de glicoconjugados en la interfase materno-fetal es de gran importancia, ya que permitirían las interacciones entre los componentes placentarios fetales y maternos, jugando un rol importante en la implantación y en el mantenimiento de la gestación (Richa et al., 1985; Cross et al., 1994; Geisert et al., 1995; Jones et al., 2000; Koncurat et al., 2004). Asimismo, alteraciones en el patrón de glicosilación podrían afectar el proceso de implantación (García et al., 2007). Intentos fallidos para obtener híbridos entre dos especies se atribuyen a una inadecuada formación de las interdigitaciones maternales; es decir, el epitelio uterino no modifica su arquitectura porque no hay una adecuada estimulación por medio del embrión ante una falla en la comunicación entre ambos tejidos (Jones et al., 2002).

Dentro de los cambios que ocurren en el endometrio uterino, es importante conocer el patrón de glicosilación que se da en el útero de la alpaca, ya que en otras especies se sabe que ciertos cambios son causantes de infertilidad y podrían estar relacionados a enfermedades reproductivas (Cipolla et al., 1998; Cobo et al., 2004; Sant'Ana et al., 2009). Por otro lado, no se disponen de estudios lectinhistoquímicos que permitan comparar el patrón de glicosilación de una hembra sometida a un tratamiento hormonal de superovulación con una hembra que no lo haya sido, así como el posible efecto en los cambios uterinos por las hormonas empleadas en el proceso de superovulación. 
El presente estudio tuvo por objetivo determinar, mediante el uso de la técnica de lectinhistoquímica, las variaciones en el patrón de glicosilación del epitelio uterino en alpacas sometidas a un tratamiento de superovulación con FSH, GnRH y hCG frente a alpacas no tratadas.

\section{Materiales y MéTodos}

El estudio se realizó en los laboratorios de Fisiología Animal y de Reproducción Animal de la Facultad de Medicina Veterinaria, Universidad Nacional Mayor de San Marcos, Lima, Perú. La colección de muestras se realizó entre los meses de julio a setiembre de 2011.

\section{Animales}

Se utilizaron 10 alpacas adultas, variedad Huacaya, con 6 a 8 años de edad, las cuales habían tenido un promedio de cinco partos previos y se encontraban destinadas para el consumo. Los animales fueron evaluados clínicamente, ninguno se encontraba en lactación y por ecografía rectal (Ecógrafo Aloka SS500, transductor Modo B de 7.5 MHz) se determinó que estaban vacías. Asimismo, su historia clínica indicaba que no habían sido sometidos a tratamientos hormonales.

\section{Tratamientos}

Los animales fueron divididos en dos grupos de cinco animales cada uno.

Sin tratamiento superovulatorio (NSO): Las alpacas fueron sacrificadas en el camal de Ñuñoa, Puno. Se confirmó que los animales no estaban gestantes y no presentaban cuerpo lúteo o folículos ováricos mayores de $4 \mathrm{~mm}$, considerándose que se encontraban en fase de anestro. Se tomaron muestras en forma inmediata al sacrificio.
Con tratamiento superovulatorio (SO): Los animales fueron sometidos a un tratamiento de superovulación para la obtención de ovocitos (Huanca et al., 2009). Se determinó la presencia de folículos $\leq 7 \mathrm{~mm}$ mediante ecografía, momento en el cual se administró $1 \mathrm{ml}$ de GnRH (Conceptal, Intervet, Holanda), equivalente a $0.042 \mathrm{mg}$ de acetato de buserelina, con el fin de inducir la ovulación (día 0 en el tratamiento). El día 2 se realizó ecografía para confirmar la ovulación en base al criterio de la desaparición del folículo previamente observado, administrándose $\mathrm{FSH}$ (Folltropin V, Bioniche, Canadá) en dosis diaria dividida cada 12 horas por 4 días, con una dosis total de $200 \mathrm{mg}$ por animal. En el día 5 se aplicó 1000 UI de gonadotropina coriónica humana (hCG) (Lutropin V, Bioniche, Canadá). El día 6 se realizó una laparatomía exploratoria para la extracción del tracto reproductivo.

\section{Toma de Muestras y Procesamiento}

Se tomaron muestras del tracto reproductivo entre aproximadamente $2 \mathrm{~cm}$ de la región media del cuerpo del útero y de la sección media de cada cuerno uterino, y se colocaron en formol buferado al 10\%. Las muestras obtenidas fueron reducidas a $0.5 \mathrm{~cm}$ de ancho por $0.5 \mathrm{~cm}$ de largo y fueron procesadas como muestras histológicas e incluidas en parafina.

Se prepararon cortes de $5 \mu \mathrm{m}$ de espesor que fueron colocados en láminas portaobjetos embebidas en Poly-L-lysina ( $\mathrm{P}$ 8920, Sigma-Aldrich), y sometidas a técnicas histoquímicas y de lectinhistoquímica (Gretchen et al., 1979; Montuenga et al., 2009).

\section{Estudio Histoquímico}

Reacción de Schiff-Acido Peryódico (PAS): Se utilizó para demostrar la presencia de polisacáridos simples y polisacáridos complejos neutros. Este método da resultado positivo ante la presencia de glucógeno, mucinas y sialomucinas. 
Cuadro 1. Distribución de áreas de estudio en el cuerpo y cuernos derecho e izquierdo del útero de alpacas

\begin{tabular}{ccccccccc}
\hline \multirow{2}{*}{$\begin{array}{c}\text { Epitelio } \\
\text { luminal }\end{array}$} & \multicolumn{6}{c}{ Epitelio glandular } & \multirow{2}{*}{$\begin{array}{c}\text { Tejido } \\
\text { conjuntivo }\end{array}$} \\
\cline { 2 - 6 } & Superficial & \multicolumn{2}{c}{ Intermedio } & Profundo & \\
\hline $\mathrm{C}^{1} \mathrm{GC}^{2}$ & $\mathrm{C}$ & $\mathrm{GC}$ & $\mathrm{C}$ & $\mathrm{GC}$ & $\mathrm{C}$ & $\mathrm{GC}$ & \\
\hline${ }^{1}$ Citoplasma & & & & & & & & \\
${ }^{2}$ Glicocáliz & & & & & & & &
\end{tabular}

Coloración Azul Alcían con pH 2.5: El grado de reactividad de esta técnica varía en función del pH del medio, lo cual permite distinguir mucopolisacáridos o polisacáridos ácidos específicos. Esta coloración se utilizó para determinar la presencia de mucinas sulfatadas ácidas débiles.

\section{Estudio Lectinhistoquímico}

Se utilizaron 14 lectinas biotiniladas del Kit I y del Kit II de lectinas de Vector Laboratories (Burlingame, CA, EEUU) y se empleó el método de avidina-biotina. En la detección y amplificación de las uniones producidas se utilizó el sistema estreptavidina peroxidasa, Horseradish peroxidase Streptavidine-RTU de Vector laboratorios (Burlingame, CA, EEUU) y para el revelado se utilizó el cromógeno 3`3 diaminobenzidina de Vector Laboratories (Burlingame, CA, EEUU). Como control positivo de cada lectina se utilizaron muestras de intestino de ratón y de alpaca, muestras en las cuales se estandarizó la técnica.

\section{Lectura de Láminas}

Las láminas trabajadas mediante histoquímica y lectinhistoquímica se leyeron en un microscopio trinocular de luz incorporada (modelo Primo Star) para exámenes en campo claro en luz transmitida con un ocular WF 10x/20, objetivos de 4x, 10x y 40x, y con una cámara digital Canon Powershot G9 de 12 megapixels montada sobre el microscopio y conectada a una computadora.
Las láminas de PAS (ácido periódicoSchiff) y de azul alcián fueron descritas mediante su reacción positiva o negativa. En las muestras procesadas mediante lectinhistoquímica se determinó la positividad ante una lectina por la coloración marrón dorado causada por el cromógeno DAB. Asimismo, en cada porción estudiada y en cada área se determinó la intensidad de marcación como: Ausencia de marcación (-), Marcación leve (+), Marcación moderada (++), Marcación fuerte $(+++)$. En casos donde la intensidad de marcación varió dentro de una misma estructura se asignaron los grados extremos (por ejemplo, $-/+++$, indicando que la intensidad de marcación varió entre negativo a fuerte). Los resultados fueron colocados en tablas de resumen explicando lo encontrado para cada lectina en cada porción y estructura.

\section{Resultados}

El epitelio que recubre el lumen endometrial era de tipo cilíndrico simple con núcleo circular basal con zonas de invaginación que forman las nuevas glándulas endometriales, las cuales fueron más numerosas en el grupo SO. Las glándulas que se ubican en las zonas más superficiales se encuentran más separadas entre sí y presentan lumen glandular de mayor diámetro. El epitelio glandular es de tipo cilíndrico simple alto con núcleo circular que por lo general se presenta en posición basal. A lo largo del estroma se encuentran vasos sanguíneos más abundantes en el grupo SO. 


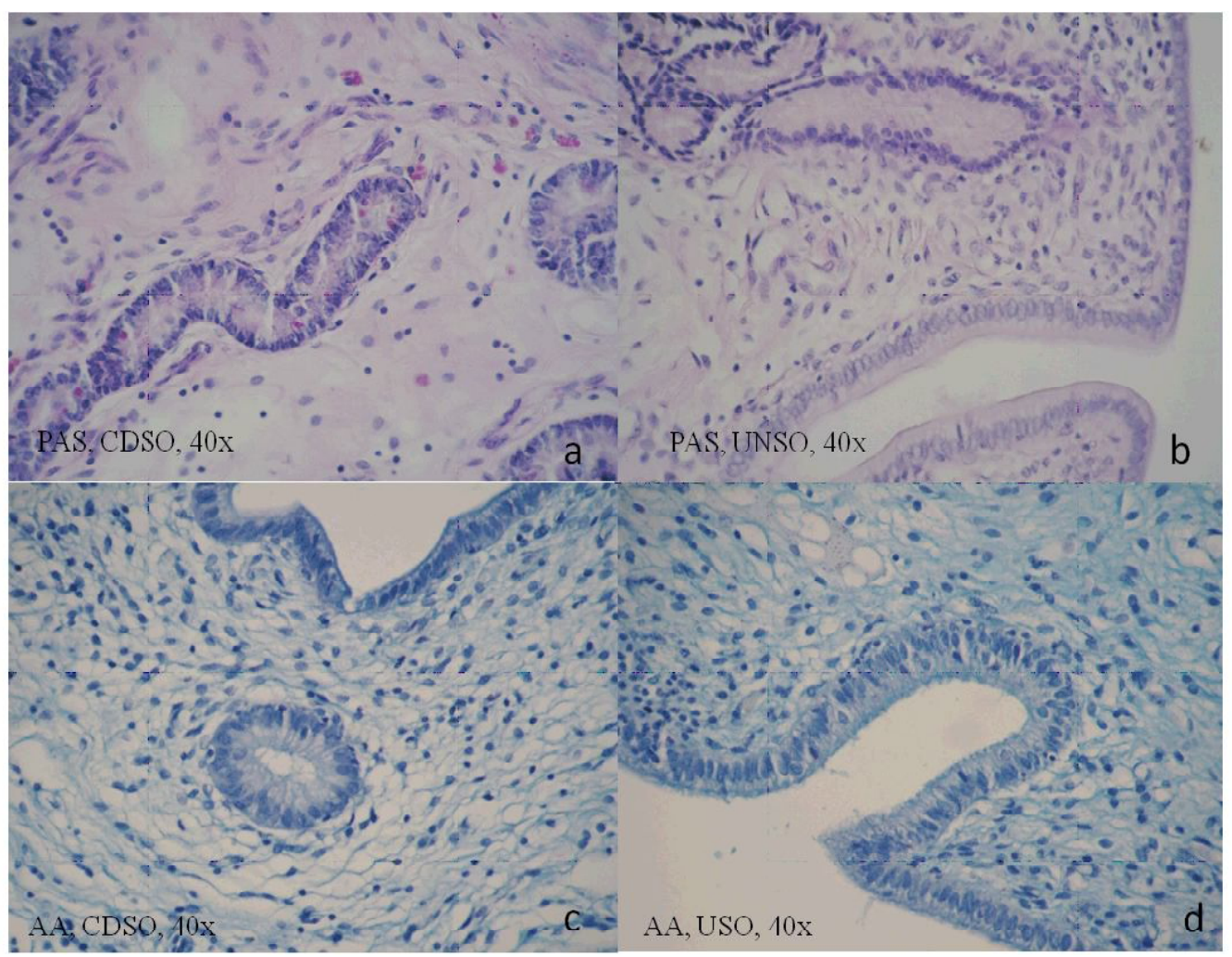

Figura 1. Estudio histoquímico (Reacción de Schiff-Ácido Peryódico - PAS, Azul Alcián pH 2.5 - $A A$ ) del útero de la alpaca. a) Coloración PAS, cuerno derecho, alpaca superovulada; b) Útero, alpaca no superovulada; c) Coloración AA, cuerno derecho, alpaca superovulada; d) Cuerpo uterino, alpaca superovulada

\section{Estudio Histoquímico}

\section{PAS}

En ambos grupos se observó que todas las estructuras del endometrio fueron PAS+, existiendo mayor intensidad en el glicocáliz del epitelio luminal endometrial y en las zonas más profundas y cercanas al tejido conjuntivo del grupo SO. También se observaron en este grupo células aisladas de algunas glándulas intermedias fuertemente positivas, principalmente en el cuerno izquierdo (Fig. 1).

\section{Azul Alcián (AB)}

La marcación con esta coloración fue similar a la observada con la técnica PAS, marcando todas las estructuras del endometrio en ambos grupos con una intensidad entre débil a moderada, resaltando la marcación en el glicocáliz del epitelio luminal y de algunas zonas del estroma, siendo mayor en intensidad en los tejidos del grupo SO (Fig. 1).

\section{Estudio Lectinhistoquímico}

Los resultados obtenidos para cada lectina se presentan en los Cuadros 2 a 4 y en la Fig. 2. Las observaciones más notables fueron:

\section{PNA}

En el cuerpo uterino del grupo SO, el glicocáliz del epitelio luminal endometrial presentó una marcación más intensa que el grupo NSO, mientras que en los cuernos uterinos 


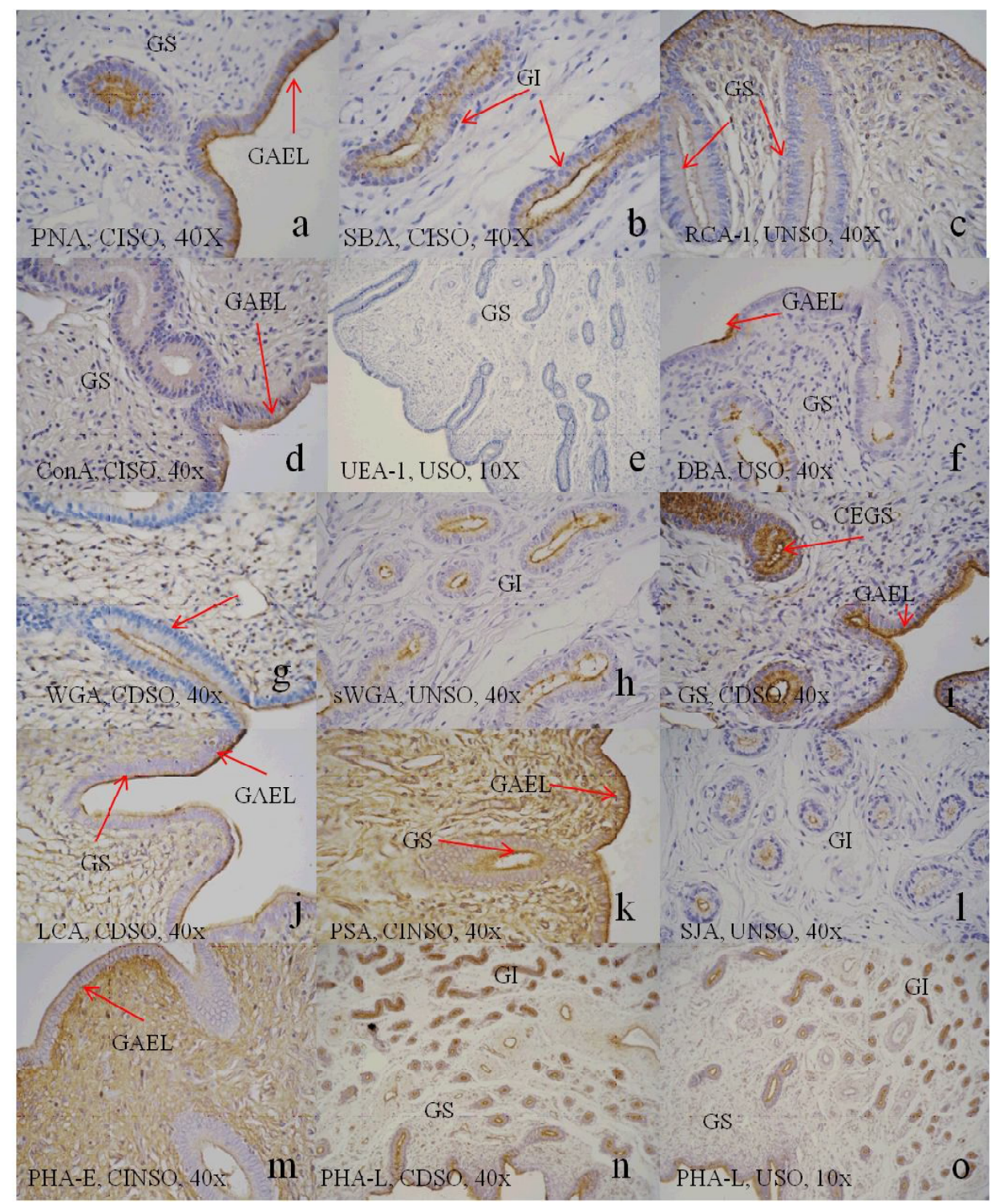

Figura 2. Marcación con lectinas en el cuerpo y cuernos uterinos de la alpaca sin tratamiento de superovulación (NSO) y en alpacas superovuladas (SO). a) Marcación con PNA en cuerno uterino izquierdo $\mathrm{SO}, \mathrm{b}$ ) marcación con $\mathrm{SBA}$ en cuerno uterino izquierdo $\mathrm{SO}, \mathrm{c}$ ) marcación con RCA-I en cuerpo uterino NSO, d) marcación con ConA en cuerno izquierdo SO, e) marcación con UEA-I en cuerpo uterino SO, f) marcación con DBA en cuerpo uterino SO, g) marcación con WGA en cuerno derecho, h) marcación con sWGA en cuerpo uterino NSO, i) marcación con GS en cuerno derecho SO, j) marcación con LCA en cuerno derecho SO, k) marcación con PSA en cuerno izquierdo NSO, 1) marcación con SJA en cuerpo uterino NSO, m) marcación con PHA-E en cuerno izquierdo NSO, n) marcación con PHA-L en cuerno derecho SO, o) marcación con PHA-L en cuerpo uterino SO. GS: glándulas superficiales, GI: glándulas intermedias, GAEL: glicocáliz apical de epitelio luminal 
Cuadro 2. Estudio lectinhistoquímico del epitelio del cuerpo uterino de alpacas no superovuladas (NSO) y bajo tratamiento superovulatorio (SO)

\begin{tabular}{|c|c|c|c|c|c|c|c|c|c|c|}
\hline \multirow{3}{*}{ Lecitina } & \multirow{3}{*}{$\mathrm{G}^{1}$} & \multirow{2}{*}{\multicolumn{2}{|c|}{ Epitelio luminal }} & \multicolumn{6}{|c|}{ Epitelio glandular } & \multirow{3}{*}{$\begin{array}{c}\text { Tejido } \\
\text { conjuntivo }\end{array}$} \\
\hline & & & & \multicolumn{2}{|c|}{ Superficial } & \multicolumn{2}{|c|}{ Intermedio } & \multicolumn{2}{|c|}{ Profundo } & \\
\hline & & $\mathrm{GA}^{2}$ & $\mathrm{C}^{3}$ & GA & $\mathrm{C}$ & GA & $\mathrm{C}$ & $\mathrm{GA}$ & $\mathrm{C}$ & \\
\hline \multirow[t]{2}{*}{$\overline{\text { PNA }}$} & $\mathrm{NSO}$ & $-1+$ & - & $++/+++$ & $-1+$ & ++ & $+/++$ & + & $-/+$ & - \\
\hline & SO & $++/+++$ & $+/++$ & $++/+++$ & $+/++$ & ++ & +/++ & + & $-/++$ & $-/+$ \\
\hline \multirow[t]{2}{*}{ SBA } & NSO & ++/+++ & $+/++$ & ++ & $+/++$ & $++/++$ & +/++ & ++ & $+/++$ & - \\
\hline & SO & $++/+++$ & + & $++/+++$ & $+/++$ & $++/+++$ & +/++ & - & - & - \\
\hline \multirow[t]{2}{*}{ RCA-1 } & NSO & +/++ & + & +/++ & $-/+$ & $+/++$ & $-/+$ & $+/++$ & + & + \\
\hline & SO & ++ & + & + & + & t/++ & + & $+/++$ & + & + \\
\hline \multirow[t]{2}{*}{ Con A } & $\mathrm{NSO}$ & $++/+++$ & + & + & + & + & + & + & $-/+$ & + \\
\hline & SO & ++ & $+/++$ & + & $-/+$ & $-/+$ & $-/+$ & - & $-/+$ & + \\
\hline \multirow[t]{2}{*}{ UEA-I } & NSO & - & - & - & - & - & - & - & - & - \\
\hline & SO & - & - & - & - & - & - & - & - & - \\
\hline \multirow[t]{2}{*}{ DBA } & $\mathrm{NSO}$ & $-/+++$ & $-/++$ & $-/+++$ & $-/++$ & - & - & - & - & - \\
\hline & SO & $-1++$ & $-/++$ & $-/++$ & $-/++$ & - & - & - & - & - \\
\hline \multirow[t]{2}{*}{ WGA } & $\mathrm{NSO}$ & $+/++$ & + & + & + & $-/+$ & $-/+$ & $-/+$ & + & + \\
\hline & SO & ++ & + & $-/+$ & - & $-/+$ & $-/+$ & $-/+$ & - & + \\
\hline \multirow[t]{2}{*}{ sWGA } & $\mathrm{NSO}$ & $-/++$ & - & $-/++$ & $-/+$ & $-/+++$ & $-/++$ & $-/+$ & - & $-/+$ \\
\hline & SO & $++/+++$ & - & $-/++$ & $-/+$ & $-/+++$ & - & $-/+$ & - & $-/+$ \\
\hline \multirow[t]{2}{*}{ GS } & NSO & +++ & H & ++ & ++ & +++ & ++ & +++ & ++ & $-/+$ \\
\hline & SO & +++ & H & + & ++ & ++ & ++ & ++ & ++ & + \\
\hline \multirow[t]{2}{*}{ LCA } & NSO & ++ & + & + & + & ++ & + & ++ & + & ++ \\
\hline & SO & +++ & H & ++ & ++ & + & + & + & + & ++ \\
\hline \multirow[t]{2}{*}{ PSA } & $\mathrm{NSO}$ & ++ & + & + & + & ++ & + & ++ & + & $++/+++$ \\
\hline & SO & $++/+++$ & $+/++$ & + & + & ++ & + & + & $-/+$ & $++/+++$ \\
\hline \multirow[t]{2}{*}{ SJA } & NSO & - & - & + & $-/+$ & + & - & - & - & $-/+$ \\
\hline & $\mathrm{SO}$ & $-/+++$ & - & $-/+++$ & - & $-/+++$ & - & - & - & $-/+$ \\
\hline \multirow[t]{2}{*}{ PHA-E } & NSO & $++/+++$ & + & + & + & ++ & + & + & $-/+$ & +++ \\
\hline & $\mathrm{SO}$ & $++/+++$ & $+/++$ & + & + & ++ & + & ++ & + & +++ \\
\hline \multirow[t]{2}{*}{ PHA-L } & NSO & $++/+++$ & + & ++ & + & +++ & ++ & +++ & ++ & $-/+$ \\
\hline & SO & +++ & H & ++ & t/++ & +++ & ++ & +++ & ++ & $-/+$ \\
\hline
\end{tabular}

la marcación fue similar a la observada en el grupo NSO.

\section{$S B A$}

La marcación a nivel de citoplasma es muy similar a la lectina PNA, siendo mayormente de tipo moderada. En las glándulas superficiales los gránulos predominan en la zona apical del citoplasma, mientras que las intermedias y profundas comienzan a dismi- nuir, al igual que en la zona glandular, disminuyendo la marcación hacia las glándulas profundas.

\section{RCA-I}

El glicocáliz del epitelio luminal del cuerpo uterino tuvo una marcación débil a moderada en NSO y moderada en SO; mientras que el glicocáliz a nivel de glándulas es de débil a moderado en ambos grupos. El cito- 
Cuadro 3. Estudio lectinhistoquímico del epitelio del cuerno uterino derecho de alpacas no superovuladas (NSO) y bajo tratamiento superovulatorio (SO)

\begin{tabular}{|c|c|c|c|c|c|c|c|c|c|c|}
\hline \multirow{3}{*}{ Lecitina } & \multirow{3}{*}{$\mathrm{G}^{1}$} & \multirow{2}{*}{\multicolumn{2}{|c|}{ Epitelio luminal }} & \multicolumn{6}{|c|}{ Epitelio glandular } & \multirow{3}{*}{$\begin{array}{c}\text { Tejido } \\
\text { conjuntivo }\end{array}$} \\
\hline & & & & \multicolumn{2}{|c|}{ Superficial } & \multicolumn{2}{|c|}{ Intermedio } & \multicolumn{2}{|c|}{ Profundo } & \\
\hline & & $\mathrm{GA}^{2}$ & $\mathrm{C}^{3}$ & GA & $\mathrm{C}$ & GA & $\mathrm{C}$ & GA & $\mathrm{C}$ & \\
\hline \multirow[t]{2}{*}{ PNA } & $\mathrm{NSO}$ & $-1+$ & $-1++$ & $++/+++$ & $-/+$ & $++/+++$ & $+/++$ & + & $-/++$ & $-1+$ \\
\hline & $\mathrm{SO}$ & $-/++$ & $+/++$ & $++/+++$ & $+/++$ & $++/+++$ & $+/++$ & + & $-/+$ & $-1+$ \\
\hline \multirow[t]{2}{*}{ SBA } & $\mathrm{NSO}$ & $-/++$ & $+/++$ & $++/+++$ & $+/++$ & $++/+++$ & $+/++$ & ++ & $-/++$ & - \\
\hline & $\mathrm{SO}$ & +++ & $-/+$ & $++/+++$ & ++ & ++ & ++ & ++ & $-/+$ & $-/+$ \\
\hline \multirow[t]{2}{*}{ RCA-1 } & $\mathrm{NSO}$ & $++/+++$ & $+/++$ & ++ & $-/+$ & + & $-/+$ & $+/++$ & $-/+$ & $+/++$ \\
\hline & $\mathrm{SO}$ & $++/+++$ & $-/+$ & $+/++$ & $-/+$ & + & $-/+$ & + & - & + \\
\hline \multirow[t]{2}{*}{ Con A } & NSO & ++ & $+/++$ & $-/+$ & $-/+$ & $-/+$ & + & $-/+$ & $-/+$ & + \\
\hline & $\mathrm{SO}$ & $++/+++$ & ++ & + & + & + & $-/+$ & $-/+$ & $-/+$ & + \\
\hline \multirow[t]{2}{*}{ UEA-I } & NSO & - & - & - & - & - & - & - & - & - \\
\hline & SO & - & - & - & - & - & - & - & - & - \\
\hline \multirow[t]{2}{*}{ DBA } & NSO & $-/++$ & $-/++$ & $-/++$ & $-/++$ & - & - & - & - & - \\
\hline & $\mathrm{SO}$ & $-/++$ & $-/++$ & $-/++$ & $-/++$ & - & - & - & - & - \\
\hline \multirow[t]{2}{*}{ WGA } & NSO & $++/+++$ & ++ & ++ & + & $+/++$ & $-/+$ & $+/++$ & $-/+$ & ++ \\
\hline & $\mathrm{SO}$ & ++ & $+/++$ & +/++ & $-/+$ & + & - & + & $-/+$ & $+/++$ \\
\hline \multirow[t]{2}{*}{ sWGA } & $\mathrm{NSO}$ & - & - & $++/+++$ & $-/+$ & $++/+++$ & $-/+$ & - & - & - \\
\hline & $\mathrm{SO}$ & $+/++$ & - & $-/++$ & - & -/++ & - & - & - & - \\
\hline \multirow[t]{2}{*}{ GS } & $\mathrm{NSO}$ & +++ & ++ & ++ & ++ & ++ & ++ & ++ & + & + \\
\hline & $\mathrm{SO}$ & +++ & ++ & +++ & ++ & +++ & ++ & +++ & ++ & + \\
\hline \multirow[t]{2}{*}{ LCA } & $\mathrm{NSO}$ & ++ & ++ & ++ & + & ++ & ++ & ++ & ++ & ++ \\
\hline & SO & +++ & ++ & ++ & ++ & ++ & + & ++ & + & ++ \\
\hline \multirow[t]{2}{*}{ PSA } & $\mathrm{NSO}$ & $++/+++$ & ++ & ++ & ++ & ++ & ++ & ++ & ++ & ++ \\
\hline & $\mathrm{SO}$ & $++/+++$ & ++ & ++ & ++ & ++ & + & ++ & + & ++ \\
\hline \multirow[t]{2}{*}{ SJA } & NSO & - & - & $-1+$ & - & $-1+$ & - & - & - & - \\
\hline & $\mathrm{SO}$ & $++/+++$ & - & $++/+++$ & - & $-/+$ & - & - & - & - \\
\hline \multirow[t]{2}{*}{ PHA-E } & $\mathrm{NSO}$ & +++ & + & ++ & $-/+$ & ++ & + & ++ & + & +++ \\
\hline & $\mathrm{SO}$ & +++ & $+/++$ & ++ & + & ++ & + & + & + & +++ \\
\hline \multirow[t]{2}{*}{ PHA-L } & $\mathrm{NSO}$ & +++ & ++ & +++ & ++ & +++ & ++ & +++ & ++ & $-/+$ \\
\hline & $\mathrm{SO}$ & $++/+++$ & ++ & +++ & $+/++$ & ++ & ++ & ++ & ++ & $-/+$ \\
\hline
\end{tabular}

plasma presenta una marcación débil en todos los epitelios y con un patrón difuso; reaccionando mayormente en la zona apical del citoplasma. En NSO hay células de las glándulas superficiales e intermedias sin marcación. La marcación es mayor en NSO que en SO en el glicocáliz del epitelio luminal y en el citoplasma del cuerno izquierdo; sin embargo, la marcación a nivel de glándulas no varía entre ambos grupos. En el cuerno derecho la marcación en citoplasma es más evidente e intensa en el grupo NSO pero a nivel glándular es similar a la observada en el cuerno izquierdo.

\section{Con A}

La marcación es más intensa en el cuerpo uterino en NSO. El glicocáliz presenta marcación fuerte en epitelio luminal y de moderado a débil en las glándulas, mientras que en SO la marcación del glicocáliz del epitelio luminal es moderada y en glándulas 
Cuadro 4. Estudio lectinhistoquímico del epitelio del cuerno izquierdo de alpacas no superovuladas (NSO) y bajo tratamiento superovulatorio (SO)

\begin{tabular}{|c|c|c|c|c|c|c|c|c|c|c|}
\hline \multirow{3}{*}{ Lecitina } & \multirow{3}{*}{$\mathrm{G}^{1}$} & \multirow{2}{*}{\multicolumn{2}{|c|}{ Epitelio luminal }} & \multicolumn{6}{|c|}{ Epitelio glandular } & \multirow{3}{*}{$\begin{array}{c}\text { Tejido } \\
\text { conjuntivo }\end{array}$} \\
\hline & & & & \multicolumn{2}{|c|}{ Superficial } & \multicolumn{2}{|c|}{ Intermedio } & \multicolumn{2}{|c|}{ Profundo } & \\
\hline & & $\mathrm{GA}^{2}$ & $\mathrm{C}^{3}$ & GA & $\mathrm{C}$ & GA & $\mathrm{C}$ & GA & $\mathrm{C}$ & \\
\hline \multirow[t]{2}{*}{ PNA } & $\mathrm{NSO}$ & $-/++$ & $-/+$ & +++ & $-/+$ & $++/+++$ & $+/++$ & $+/++$ & $-/++$ & $-/+$ \\
\hline & $\mathrm{SO}$ & ++ & $+/++$ & ++ & $+/++$ & $+/++$ & $+/++$ & $+/++$ & + & $-/++$ \\
\hline \multirow[t]{2}{*}{ SBA } & NSO & $++/+++$ & $+/++$ & +++ & ++ & $++/+++$ & $+/++$ & $+/++$ & + & - \\
\hline & SO & $++/+++$ & ++ & $++/+++$ & ++ & $++/+++$ & ++ & + & + & - \\
\hline \multirow[t]{2}{*}{ RCA-1 } & NSO & +++ & $+/++$ & $+/++$ & $-/+$ & + & $-/+$ & + & - & $+/++$ \\
\hline & $\mathrm{SO}$ & ++ & $-/++$ & $+/++$ & + & + & $-/+$ & $+/++$ & $-/+$ & $+/++$ \\
\hline \multirow[t]{2}{*}{ Con A } & $\mathrm{NSO}$ & +++ & ++ & + & + & + & + & + & $-/+$ & + \\
\hline & $\mathrm{SO}$ & ++ & ++ & + & + & + & + & + & + & + \\
\hline \multirow[t]{2}{*}{ UEA-I } & NSO & - & - & - & - & - & - & - & - & - \\
\hline & $\mathrm{SO}$ & - & - & - & - & - & - & - & - & - \\
\hline \multirow[t]{2}{*}{ DBA } & $\mathrm{NSO}$ & $-/++$ & $-/+$ & $-/+++$ & $-1++$ & - & - & - & - & - \\
\hline & $\mathrm{SO}$ & $-/++$ & $-1++$ & $-/++$ & $-/+$ & - & - & - & - & - \\
\hline \multirow[t]{2}{*}{ WGA } & NSO & $++/+++$ & $+/++$ & $+/++$ & + & + & $-/+$ & + & $-/+$ & + \\
\hline & $\mathrm{SO}$ & ++ & + & $+/++$ & - & + & + & $+/++$ & $-/+$ & $+/++$ \\
\hline \multirow[t]{2}{*}{ sWGA } & NSO & - & - & $-/++$ & - & $-/+++$ & - & - & - & $-/+$ \\
\hline & $\mathrm{SO}$ & $-/+++$ & - & $-/++$ & - & $-/+++$ & - & $-/+$ & - & $-/+$ \\
\hline \multirow[t]{2}{*}{ GS } & NSO & +++ & ++ & ++ & ++ & ++ & ++ & ++ & ++ & + \\
\hline & $\mathrm{SO}$ & +++ & ++ & +++ & ++ & +++ & ++ & ++ & ++ & + \\
\hline \multirow[t]{2}{*}{ LCA } & $\mathrm{NSO}$ & ++ & ++ & ++ & ++ & ++ & + & ++ & + & ++ \\
\hline & $\mathrm{SO}$ & ++ & + & ++ & + & + & + & + & + & + \\
\hline \multirow[t]{2}{*}{ PSA } & NSO & ++ & + & ++ & $+/++$ & + & + & + & $-/+$ & - \\
\hline & $\mathrm{SO}$ & $++/+++$ & + & $+/++$ & ++ & + & + & + & - & ++ \\
\hline \multirow[t]{2}{*}{ SJA } & NSO & - & - & $-/+$ & - & $-/+$ & - & - & - & - \\
\hline & SO & - & - & $-/+$ & - & $-/+$ & - & - & - & - \\
\hline \multirow[t]{2}{*}{ PHA-E } & NSO & +++ & + & ++ & + & $+/++$ & + & + & $-/+$ & $++/+++$ \\
\hline & $\mathrm{SO}$ & ++ & + & +/++ & + & ++ & + & + & + & +++ \\
\hline \multirow[t]{2}{*}{ PHA-L } & $\mathrm{NSO}$ & ++ & $+/++$ & $++/+++$ & $+/++$ & $++/+++$ & $+/++$ & $++/+++$ & $-/+$ & $-/+$ \\
\hline & $\mathrm{SO}$ & +++ & ++ & +++ & ++ & ++ & ++ & ++ & ++ & $+/++$ \\
\hline
\end{tabular}

es débil, desapareciendo conforme las glándulas se van haciendo más profundas. En el citoplasma de las glándulas la marcación es escasa y débil en NSO, mientras solo ocurre en algunas células en SO. El patrón de unión a esta lectina es similar para ambos grupos en el cuerno izquierdo, mientras que a nivel del epitelio luminal del cuerno derecho es más intensa en SO.
$U E A-I$

No se detectaron sitios de unión a esta lectina.

$D B A$

El patrón de marcación de esta lectina se repite en cuerpo y cuernos uterinos de ambos grupos, aunque en mayor número en NSO. No se observaron sitios de unión en glándulas profundas e intermedias, mientras 
que en el epitelio luminal y en glándulas superficiales pocas células reaccionan con esta lectina.

\section{WGA}

El estroma del cuerpo uterino reacciona débilmente con esta lectina, mientras que en los cuernos uterinos marca débilmente en NSO y entre débil a moderado en SO. El glicocáliz se une de manera débil a moderada en epitelio luminal y glándulas superficiales, tornándose negativa conforme las glándulas se profundizan. La marcación en epitelio luminal es similar en ambos grupos. En el cuerno derecho, la marcación es moderada en ambos grupos a nivel de epitelio luminal, mientras que es mayor en NSO a nivel de glándulas. En el cuerno izquierdo la marcación es más intensa a nivel del epitelio luminal en NSO, mientras que en NSO la unión del glicocáliz del epitelio glandular a esta lectina es débil y el citoplasma presenta algunas zonas que marcan débilmente; asimismo, el glicocáliz de las células glandulares en SO presenta una marcación entre débil y moderada, mientras que el citoplasma se presenta negativo en las glándulas superficiales y en algunas células de las glándulas profundas.

\section{sWGA}

Esta lectina presenta un patrón de unión singular, pues hay alternancia de células marcadas y no marcadas en glándulas; asimismo, el glicocáliz se presenta como una capa muy fina. En NSO, el glicocáliz varía de negativo a moderada o fuerte en glándulas, mientras que el citoplasma varía de negativo a moderado, siendo esta marcación similar a lo observado en SO. Los cuernos uterinos de NSO no presentan marcación en epitelio luminal pero es de moderada a fuerte en glándulas.

\section{$G S-1$}

El patrón de marcación en ambos grupos fue muy similar. En los tres tejidos de ambos grupos se observó una marcación moderada con gránulos en todo el citoplasma, tanto a nivel de células glandulares como del epitelio luminal del endometrio; mientras que a nivel del estroma, esta lectina reaccio- na débilmente, observándose zonas negativas. En el caso de las glándulas, el citoplasma se une de manera moderada a esta lectina; y en el caso del glicocáliz, la intensidad es mayor en el cuerpo uterino del NSO y menor en los cuernos uterinos en comparación con SO.

\section{$L C A$}

En el caso de NSO, la unión de esta lectina al glicocáliz del epitelio luminal es moderada, así como en el citoplasma a nivel de la zona apical donde se logran distinguir mayor cantidad de gránulos. En el caso de SO, el epitelio luminal del endometrio del cuerpo uterino y cuerno derecho presenta un glicocáliz y citoplasma con marcación fuerte y moderada, respectivamente.

\section{PSA}

La marcación es moderada en los tres tejidos de ambos grupos a nivel de epitelio luminal, aunque la marcación es más intensa en SO. A nivel del citoplasma, la marcación es moderada en cuerno derecho y cuerpo uterino, perdiéndose la intensidad en el cuerno izquierdo de ambos grupos. La marcación es de moderada en glicocáliz y débil en citoplasma en las glándulas del cuerpo uterino de ambos grupos. La marcación tiende a ser más intensa en las estructuras que componen las glándulas del cuerno derecho y del cuerpo uterino en NSO, mientras que el epitelio glandular presenta marcación de menor intensidad en el cuerno izquierdo.

\section{SJA}

El citoplasma de las células del epitelio luminal y glandular del tejido endometrial no se unió a esta lectina, a excepción de algunas glándulas superficiales e intermedias donde hubo marcación débil. En los tres tejidos de NSO, el glicocáliz presenta marcación débil pero no uniforme a nivel de las glándulas superficiales e intermedias, y negativo a nivel de glicocáliz de epitelio luminal endometrial y de glándulas profunda; en cambio, en el cuerno derecho y cuerpo uterino de SO, se observa marcación más intensa en el glicocáliz de glándulas superficiales e intermedias, aun- 
que sigue siendo negativo en glándulas profundas.

\section{PHA-E}

El estroma se marca fuertemente en todos los casos y es similar entre ambos grupos. La unión a esta lectina en el cuerpo uterino es entre moderada y fuerte, mientras que en las glándulas, la marcación en glicocáliz es moderada y en citoplasma es débil. Este patrón se repite en el cuerno derecho, siendo más intensa la marcación en algunas estructuras de SO. En el cuerno izquierdo, el patrón es similar entre ambos grupos, aunque a nivel del epitelio luminal y glandular la intensidad se pierde ligeramente en SO.

\section{PHA-L}

El patrón de marcación de esta lectina es similar en el cuerno derecho y cuerpo uterino, presentando marcación moderada a fuerte en glicocáliz de epitelio luminal endometrial y de epitelio glandular. En el cuerno derecho de SO hay una disminución en la marcación a nivel del glicocáliz de las glándulas intermedias y profundas y del epitelio luminal; mientras que a nivel del citoplasma la marcación es moderada y de manera granular en todos los casos. Asimismo, en este grupo hay una disminución en la intensidad de la marcación a nivel del glicocáliz del epitelio de glándulas intermedias y profundas del cuerno izquierdo; siendo lo contrario a nivel del glicocáliz del epitelio luminal endometrial y de las glándulas superficiales y del citoplasma. La marcación en estroma es débil y en algunas zonas del endometrio en los tres tejidos de ambos grupos.

\section{Discusión}

La reacción PAS positiva encontrada en todos los tejidos de ambos grupos indica la presencia de glicopolisacáridos neutros, y el azul alcián positivo de $\mathrm{pH}$ ácido 2.5 indica la presencia de mucinas sulfatadas ácidas débiles (mayor parte de mucinas), coincidiendo con resultados de otros estudios (Walter y
Bavdek,1997; Schencke et al., 2004; Sanchis et al., 2009). La mayor intensidad de marcación mostrada en ambas técnicas en tejidos del grupo SO podría evidenciar una mayor actividad secretora del tejido endometrial, producto de las hormonas utilizadas en la superovulación.

La marcación con la lectina PNA en cuerpo uterino y cuerno derecho a nivel de epitelio luminal endometrial en NSO es similar a la encontrada por Munson et al. (1989) en tejido uterino de vacas no preñadas en fase luteal; mientras que la unión al epitelio luminal endometrial de los tres tejidos en SO y en cuerno izquierdo en NSO es similar a resultados en útero de mujeres sometidas a un tratamiento de superovulación con FHS y hCG y en mujeres que no tuvieron ninguna terapia hormonal y que no estaban gestantes (Gheri et al., 1998).

La marcación de la lectina SBA a nivel de glicocáliz del epitelio luminal y epitelio glandular de cuerno izquierdo y útero de ambos grupos de alpacas, así como en el cuerno de SO es similar a la encontrada en útero de cerdas en estro y metaestro, de perras en anestro y de mujeres sometidas a un tratamiento de superovulación (Zhou et al., 1994; Gheri et al., 1998; Leitner et al., 2003; Sant'Ana et al., 2009). Asimismo, la diferencia detectada en la marcación a nivel del glicocáliz del epitelio luminal endometrial del cuerno derecho de NSO es igual a la detectada en útero de perras con piometra y en aquellas en fase tardía de metaestro (Leitner et al., 2003).

No hay mucha diferencia en la marcación con la lectina RCA-1 entre ambos grupos de alpacas. Estos resultados se asemejan a aquellos en cerdas en fase luteal y fase folicular, e inclusive en útero de cerdas con hiperplasia endometrial quística y con endometritis (Sant'Ana et al., 2009). En mujeres se puede observar un efecto del tratamiento de superovulación en la unión de la lectina ConA, ya que presentan gránulos citoplasmáticos en el epitelio glandular y en 
epitelio luminal endometrial, mientras que en mujeres no tratadas no se observan en este último tipo de células (Gheri et al., 1998).

Los hallazgos en la marcación de la lectina UEA-1 son iguales a los encontrados por Munson et al. (1989) en vacas con más de 80 días de gestación, por Gheri et al. (1998) en mujeres sin tratamiento hormonal previo, por Lee et al. (1983) en ratas no preñadas y por Leitner et al. (2003) en perras en metaestro temprano. Gheri et al. (1998) encontraron en 3 de 12 mujeres sometidas a un tratamiento de superovulación una unión débil a esta lectina a nivel de glicocáliz del epitelio luminal endometrial y unión moderada a nivel del citoplasma células de algunas glándulas. Marcación similar se presenta en conejas en estro y pseudopreñez, aunque con un patrón no uniforme (Anderson et al., 1986).

En ninguna especie se encontró un patrón de marcación similar con la lectina DBA al de los tres tejidos de ambos grupos (Munson et al., 1989; Gheri et al., 1998; Sant'Ana et al., 2009), aunque se presentó a nivel de células linfoides en estroma uterino de vaca (Munson et al., 1989). Se sabe que el glicoconjugado de unión a la lectina DBA $(\alpha-D-G a l N a c)$ posee propiedades de antiadhesividad, de allí que se le encuentre en muy escasa cantidad en el cuerpo y cuernos uterinos de la alpaca (Hoffman et al., 1998), y que, asimismo, las hormonas utilizadas en la superovulación disminuyen la expresión de este azúcar en estas estructuras, dado que los sitios de unión a esta lectina fueron más escasos en $\mathrm{SO}$ comparados con NSO.

La lectina WGA tiene afinidad por Nacetil-glucosamina y ácido siálico, mientras que su derivado succinilado sWGA se une específicamente a $\mathrm{N}$-acetilglucosamina y a sus oligómeros (Monsigny et al., 1979). En este estudio, la falta de unión de sWGA en las zonas donde se presentó marcación a la lectina WGA sostiene la presencia de ácido siálico a ese nivel (Anderson et al., 1986), el cual se cree está presente como parte estructural. La N-acetil-glucosamina está relacionada con el ácido hialurónico, el cual participa en la migración celular, y que podría actuar en las células epiteliales que pasarán a formar las nuevas glándulas uterinas (Alberts et al., 1989).

Munson et al. (1989) encontraron que la marcación con la lectina LCA en vacas en fase luteal es débil a nivel del glicocáliz del epitelio luminal endometrial y del glandular, pero moderada a nivel del citoplasma del epitelio luminal endometrial. Asimismo, en útero de ratas vírgenes de 6-8 semanas de edad no hubo unión con esta lectina a nivel de ningún epitelio y solo a nivel del estroma (Lee et al., 1983) como en el presente estudio. Jones et al. (2002) presentan resultados similares en muestras de placenta de alpacas en gestación temprana y avanzada.

El patrón de unión de PHA-E encontrado en alpacas difiere del que se presenta en vacas en fase luteal donde la marcación es débil a nivel epitelio luminal endometrial y glandular (Munson et al., 1989), así como en ratas de 6-8 semanas de edad no gestantes que presentan unión débil en algunas zonas del glicocáliz, tanto del epitelio luminal endometrial como del glandular (Lee et al., 1983).

La lectina PHA-L se une débilmente a nivel de todo el endometrio en ratas no gestantes y en ratas con 12 días de gestación (Lee et al., 1983). La marcación es muy débil en la gestación temprana de la alpaca y se hace negativa en la gestación tardía (Jones et al., 2002).

Si bien no se conoce el rol que ejercen los glicoconjugados que se unen a las lectinas, la determinación de patrones de glicosilación para las lectinas PNA, RCA-I, Con A, DBA, WGA, sWGA, GS, LCA, SJA y PHA-L, donde se observaron diferencias entre grupos, sugiere que el tratamiento hormonal influenció en la expresión de los glicoconjugados que se unen a estas lectinas; a través del efecto de 
la FSH, GnRH y hCG así como el del estrógeno proveniente de los folículos generados por el tratamiento de superovulación sobre la proliferación del tejido endometrial; como se ha comprobado en estudios previos en mamíferos (Anderson et al., 1986; Pentecost y Teng, 1987; Gheri et al., 1998; Leitner et al., 2003).

Las lectinas SBA, RCA-I, UEA, DBA, WGA, sWGA, GS, PHA-E y PHA-L presentan patrones de marcación similares para ambos cuernos y entre ambos grupos. Las diferencias en el patrón de marcación de la lectina PNA y SJA entre el cuerno derecho y el izquierdo serían como resultado del cambio hormonal generado por el tratamiento de superovulación; mientras que las diferencias por la lectina Con A entre ambos cuernos ocurren solamente en NSO. Las lectinas LCA, PSA presentan un patrón de marcación diferente entre el cuerno derecho y el cuerno izquierdo en ambos grupos, lo que podría sugerir algún rol en el porcentaje mayoritario de gestaciones en el cuerno izquierdo.

La marcación de células linfoides con las lectinas SJA, GS, y DBA en cuerpo uterino y cuernos uterinos de la alpaca es un hallazgo normalmente presente en el tejido uterino de otras especies y ocurre porque la mucosa del tracto reproductor femenino está normalmente expuesta a microorganismos patógenos (Munson et al., 1989; Del Rey, 1992). En el presente trabajo se observó mayor cantidad de células inmunológicas en el endometrio de cuerpo y cuernos uterinos de alpacas SO, lo cual concuerda con otras observaciones en útero de ratones, donde se comprobó la influencia de las hormonas sexuales en el aumento de la población de células inmunológicas a nivel del endometrio uterino (Wira y Stern, 1992).

\section{Conclusiones}

- El patrón de glicosilación en el endometrio del cuerpo y cuernos uterinos es diferente, especialmente a nivel de cuerno izquierdo, lo que podría determinar que la implantación en la alpaca ocurra mayormente a este nivel.

- El tratamiento hormonal utilizado en el proceso de superovulación al que fueron sometidas las alpacas alteró el patrón de glicosilación tanto en el cuerpo como en los cuernos uterinos.

\section{Literatura Citada}

1. Alberts B, Bray D, Lewis J, Raff M, Roberts K, Watson J. 1989. Molecular Biology of the Cell. $2^{\text {nd }}$ ed. New York: Garland. 200 p.

2. Anderson T, Olson G, Hoffman L. 1986. Stage-specific alterations in the apical membrane glycoproteins of endometrial epithelial cells related to implantation in rabbits. Biol Reprod 34: 701-720.

3. Cipolla A, Paolicchi F, Poso M, Morsella C, Casaro C, Massone A, Villegas $R$, et al. 1998. Lectin-binding sites in uterus and oviduct of normal and Campylobacter fetus subspecies veneralis-infected heifers. Eur J Histochem 42: 63-70.

4. Cobo E, Campero C, Gimeno E, Barbeito C. 2004. Lectin binding patterns and inmunohistochemical antigen detection in the genitalia Tritrichomonas foetus-infected heifers. J Comp Pathol 131: 127-134.

5. Cross J, Werb Z, Fisher S. 1994. Implantation and the placenta: key pieces of the development puzzle. Science 266: 1508-1518.

6. Del Rey P. 1992. Importancia inmunitaria de las mucosas en la reproducción humana. En: Acosta G, Cruz M (eds). Inmunología de las mucosas. $3^{\mathrm{a}}$ ed. México: Atelier Producciones. p 119-126.

7. Fernández Baca S, Hansel W, Novoa C. 1970. Corpus luteum function in the alpaca. Biol Reprod 3: 252-261.

8. García J, Arcila X, Cano J, Isaza V, Tovar A, Valencia A, Moreno F. 2007. Expresión de azúcares endometriales y 
del oviducto durante el ciclo reproductivo de la coneja. Rev Chi Obstet Ginecol 72: 176-181.

9. Geisert R, Dixon M, Pratt T, Schmitt R, Lessley B, McCann J. 1995. Isolation and characterization of a 30$\mathrm{kDa}$ endometrial glycoprotein synthesized during the estrous cycle and early pregnancy of the pig. Biol Reprod 53: 1038-1050.

10. Gheri G, Gheri S, Brykl, G, Taddei G, Borri P, Noci I. 1998. Lectin binding in the human endometrium in early luteal phase following controlled ovarian hyperstimulation. Histol Histopathol 13: 737-742.

11. Hoffman L, Olson G, Carson D, Chilton B. 1998. Progesterone and implanting blastocysts regulate Muc1 expression in rabbit uterine epithelium. Endocrinology 139: 266-271.

12. Huanca W, Cordero A, Huanca T, Cardenas O, Adams GP, Ratto MH. 2009. Ovarian response and embryo production in llamas treated with equine chorionic gonadotropin alone or with a progestin-releasing vaginal sponge at the time of follicular wave emergence. Theriogenology 72: 803-808.

13. Jones $C$, Wooding F, Abd-Elnaeim $M$, Leiser R, Dantzer V, Stoddart R. 2000. Glycosylation in the nearterm epitheliochorial placenta of the horse, donkey and camel: a comparative study of interbreeding and non-interbreeding species. $\mathrm{J}$ Reprod Fert 118: 397-405.

14. Jones C, Abd-Elnaeim M, Bevilacqua E, Oliveira L, Leiser R. 2002. Comparison of uteroplacental glycosylation in the camel (Camelus dromedarius) and alpaca (Lama pacos). Reproduction 123: 115-126.

15. Koncurat M, Merkis C, Zubeldía D, Chanique A, Cristofolini A, Franchino M, Abate Cano L, et al. 2004. Detección de glicoconjugados en la placenta porcina de diferentes estadíos gestacionales. Int J Morphol 22: 56-57.

16. Lee M, Wu T, Wan Y, Damjanov I. 1983. Pregnancy-related changes in the mouse oviduct and uterus revealed by differential binding of fluoresceinated lectins. Histochemistry 79: 365-375.

17. Leitner M, Aurich E, Galabova G, Aurich C, Walter I. 2003. Lectin binding patterns in normal canine endometrium and in bitches with pyometra and cystic endometrial hyperplasia. Histol Histopathol 18: 787795.

18. Monsigny M, Sene C, Obrenovitch A, Roche A, Delmotte F, Boschette E. 1979. Properties of succinylated wheat germ agglutinen. Eur J Biochem 98: 39-45.

19. Montes M, Quicaño I, Quispe R, Quispe E, Alfonso L. 2008. Quality characteristics of Huacaya alpaca fibre produced in the Peruvian Andean Plateau region of Huancavelica. Spanish J Agric Res 6(1): 33-38.

20. Munson L, Kao J, Schlafer D. 1989. Characterization of glycoconjugates in the bovine endometrium and chorion by lectin histochemistry. J Reprod Fert 87: 509-517.

21. Novoa C. 1991. Fisiología de la reproducción de la hembra. En: Fernández Baca $S$ (ed). Avances y perspectivas del conocimiento de los camélidos sudamericanos. Santiago de Chile: Oficina Regional de la FAO para América Latina y el Caribe. p 91-107.

22. Pentecost B, Teng C. 1987. Lactotransferrin is the major estrogen inducible protein of mouse uterine secretions. J Biol Chem 262: 1013410139.

23. Psychoyos A. 1986. Uterine receptivity for nidation. Ann NYAcad Sci 476: 36-42.

24. Quispe E, Rodríguez T, Iñiguez L, Mueller J. 2009. Producción de fibra de alpaca, llama, vicuña y guanaco en Sudamérica. Anim Gen Resour Info 45: 1-14.

25. Richa J, Damsky C, Buck C, Knowless $B$, Solter D. 1985. Cell surface glicoproteins mediate compaction, trophoblast attachment, and endoderm formation during early mouse development. Dev Biol 108: 513-521. 
26. Sant'Ana F, Nascimento E, Laube P, Barbeito C. 2009. Lectin-binding sites on the normal and pathologic uterus of sows. Reprod Dom Anim 44: 889-893.

27. Sanchis E, Merkis C, Koncurat M. 2009. Detección de glicoconjugados en las vellosidades placentarias porcinas de diferentes períodos gestacionales. REDVET 10(12): 1-12.

28. Schencke C, Del Sol M, Rojas M. 2004. Relación útero-embrionaria y su variación morfológica durante el periodo implantacional en conejo. Int $\mathbf{J}$ Morphol 22: 313-322.

29. Tachi S, Tachi C, Lindner H. 1970. Ultrastructural features of blastocyst attachment and trophoblastic invasion in the rat. J Reprod Fert 21: 37-56.
30. Walter I, Bavdek S. 1997. Lectin binding patterns of porcine oviduct mucosa and endometrium during the oestrous cycle. J Anat 190: 299-307.

31. Whyte A, Allen W. 1985. Equine endometrium at pre-implantation stages of pregnancy has specific glycosylated regions. Placenta 6: 537-542.

32. Zhou Z, Deng Z, Chen J. 1994. Characterization of glycoconjugates in the endometrium of the miniature pig during different reproductive periods. J Reprod Fert 100: 417-423.

33. Wira C, Stern J. 1992. Endocrine regulation of the mucosal immune system in the female reproductive tract. In: Pasqualini J, Scholler R (eds). Mucosal immunology. San Francisco: Elsevier. p 57-68. 